\title{
The Permeability of Ablative Materials Under Rarefied Gas Conditions
}

\author{
Craig White ${ }^{\mathrm{a}, *}$, Thomas J. Scanlon ${ }^{\mathrm{b}}$, Richard E. Brown ${ }^{\mathrm{b}}$ \\ ${ }^{a}$ School of Engineering, University of Glasgow, Glasgow G12 8QQ, UK \\ ${ }^{b}$ Department of Mechanical and Aerospace Engineering, University of Strathclyde, \\ Glasgow G1 1XJ, UK
}

\begin{abstract}
Numerical meshes of both cork and carbon fibre ablative materials in their virgin and pyrolised states, with realistic porosity and tortuosity, have been created from micro-computed tomography $(\mu \mathrm{CT})$ scans. The porosity of each material has been calculated from the $\mu \mathrm{CT}$ scans and used to extract smaller representative sample volumes to perform numerical simulations on. Direct simulation Monte Carlo simulations of rarefied gas flow through these materials have been performed to find the permeability of each material to argon gas and to a gas mixture. The method has been validated by comparing the measured permeability for a Berea sandstone material to previously published experimental values. For the specific pressure conditions investigated here, the cork-phenolic material becomes around ten mores permeable after being pyrolised, while the carbon-phenolic material only becomes five times more permeable than its virgin form. The permeability to the gas mix-
\end{abstract}

\footnotetext{
*Corresponding author

Email addresses: craig.white.2@glasgow.ac.uk (Craig White), tom.scanlon@strath.ac.uk (Thomas J. Scanlon), richard.brown@strath.ac.uk (Richard E. Brown)
} 
ture is found to be greater than to argon for most of the samples, showing the importance of choosing the correct gas for rarefied permeability studies. The form of the pressure and Mach number profiles through the materials is indepedent of the applied pressure gradient.

Keywords: permeability, rarefied gas, ablation, re-entry, CT scan, DSMC

\section{Nomenclature}

\begin{tabular}{|c|c|}
\hline$A$ & cross-sectional area, $\mathrm{m}^{2}$ \\
\hline$b$ & Klinkenberg slip correction factor, $\mathrm{Pa}$ \\
\hline c & molecular velocity vector, m/s \\
\hline$c_{r}$ & relative speed, m/s \\
\hline$D$ & characteristic length, $\mathrm{m}$ \\
\hline$d$ & molecular diameter, $\mathrm{m}$ \\
\hline $\mathbf{F}$ & force vector, $\mathrm{N}$ \\
\hline$f$ & normalised velocity distribution function \\
\hline $\mathbf{J}$ & binary collision integral \\
\hline$K n$ & Knudsen number \\
\hline$k$ & permeability, $\mathrm{m}^{2}$ \\
\hline$k_{0}$ & permeability to a liquid, $\mathrm{m}^{2}$ \\
\hline$L$ & length, m \\
\hline$M$ & molar weight, $\mathrm{kg} / \mathrm{mol}$ \\
\hline$m$ & molecular mass, kg \\
\hline$\dot{m}$ & mass flowrate, $\mathrm{kg} / \mathrm{s}$ \\
\hline$n$ & number density, $\mathrm{m}^{-3}$ \\
\hline$P$ & pressure, $\mathrm{Pa}$ \\
\hline$\dot{Q}$ & volume flowrate, $\mathrm{m}^{3} / \mathrm{s}$ \\
\hline
\end{tabular}


$r$

T

$X$

$Z_{\text {rot }}$

Greek

$\Theta_{v}$

$\lambda$

$\mu$

$\xi$

$\rho$

$\Omega$

$\omega$

Superscripts

*

Subscripts

in

out position vector, $\mathrm{m}$

temperature, $\mathrm{K}$

molar fraction

rotational relaxation number

characteristic vibrational temperature, $\mathrm{K}$

mean free path, $\mathrm{m}$

viscosity, $\mathrm{kg} \mathrm{s}^{-1} \mathrm{~m}^{-1}$

molecular cross-section, $\mathrm{m}^{2}$

mass density, $\mathrm{kg} / \mathrm{m}^{3}$

solid angle, rad

viscosity exponent

post-collision properties

inlet properties

outlet properties

\section{Introduction}

During an atmospheric entry, a space vehicle is subjected to extremely high heating loads due to the temperatures of the gas in the shock layer immediately upstream of the vehicle. Historically, a single-use ablative heat shield was used as a thermal protection system (TPS) to ensure that the vehicle could survive this extreme heating load; however, as space access developed mainly to low Earth orbit missions with the introduction of space 
stations and the Space Shuttle, the research focus switched to re-usable thermal protection systems. Recent missions have ventured further than low Earth orbit, and have therefore required more robust heat shields. For this reason, a shift back to ablative heat shields has been observed recently. For example, the Stardust sample return mission [1] used a phenolic impregnated carbon ablative heat shield [2].

During the ablation process, the heat load applied to the material is dispersed first through the melting of a phenolic filler resin that results in the production of pyrolysis gases; in addition to absorbing energy through the melting process, the pyrolysis gas is blown in to the boundary layer and reduces the heat flux at the surface by preventing the hot gas from the shock layer from penetrating to the surface. In addition to pyrolysis gas blowing, ablation takes place, which is the degradation of material through thermal, chemical, and mechanical processes. The heat shield is damaged under the extreme aero-thermo-mechanical loads, but the spacecraft itself remains intact, protecting the payload and humans from the harsh re-entry environment. Besides the safety aspect, it is also important to ensure that the mission is as cost-effective as possible; one aspect of this can be designing a TPS that is as small and light as possible so that more payload can be carried.

The ablation process is highly complex and multidisciplinary, and so understanding it well enough to design optimised heat shields remains an engineering challenge. One key area is the flow of the pyrolysis gas products out of the material and into the boundary layer. Ablative heat shields are made of a preform (e.g. carbon fibres) that is filled with a phenolic resin. The 
pyrolysis gas blowing can then be thought of as the flow of a compressible fluid through a porous material, which is driven by a pressure gradient between the base of the pyrolysis zone and the stagnation pressure at the outer edge of the heat shield. Three-dimensional effects may also be important, with flow also escaping from the sides of an ablative material [3]. The property that defines how easily a fluid can traverse a porous medium is know as permeability. It is important when developing predictive tools for ablative materials to have a good understanding of how the permeability of the material changes with temperature, gas pressure, and the degree of material breakdown.

The average permeability $k\left(\mathrm{~m}^{2}\right)$ of a porous medium to a compressible fluid is given by [4]:

$$
k=\frac{\dot{Q} \mu L}{A} \frac{2 P_{\text {out }}}{P_{\text {in }}^{2}-P_{\text {out }}^{2}},
$$

where $\dot{Q}$ is the outlet volumetric flow rate of the fluid from the material, $\mu$ is the fluid viscosity, $L$ is the length of the sample in the flow direction, $A$ is the cross-sectional area (ignoring the voids introduced by porosity), $P_{\text {in }}$ is the inlet pressure, and $P_{\text {out }}$ is the outlet pressure. Equation (1) is only valid for one dimensional cartesian flow, with every parameter held constant. The permeability of a medium to gases becomes progressively greater than that of its liquid permeability as the gas density decreases, a phenomenon that is know as the Klinkenberg effect [5], and is attributed to non-equilibrium gas effects that are well known in the rarefied gas field. As the mean free path $\lambda$ (the average distance a gas atom/molecule travels before undergoing a collision) of the gas approaches the mean pore diameter $D$ in a porous medium, non-equilibrium effects become important and lead to 
the gas 'slipping' across the surface [6], which results in enhanced transport of mass compared to a liquid. The Knudsen number,

$$
K n=\lambda / D,
$$

is used as a measure of gas rarefaction. Figure 1 illustrates the different Knudsen number regimes.

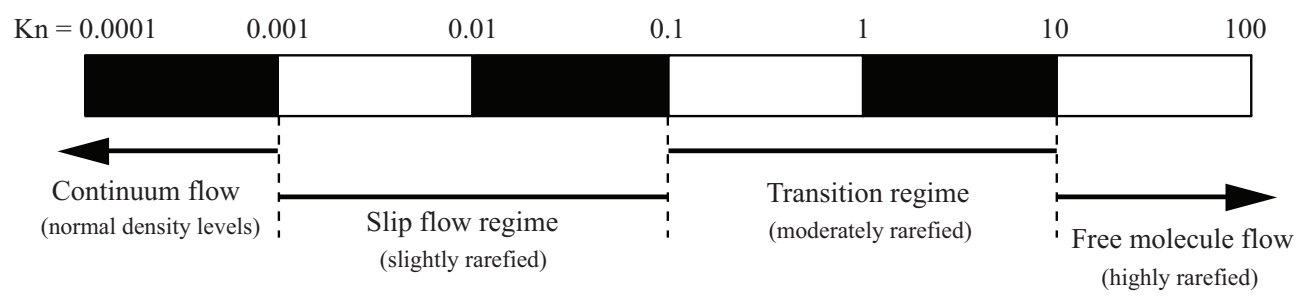

Figure 1: Knudsen number regimes, adapted from Reference [7].

As has previously been discussed by Lachaud et al. [8], if the pore sizes in the internal structure of a heat shield are on the order of micrometres, the gas flow can fall within the transition Knudsen number regime due to the small length scale and the relatively small magnitude of the stagnation pressures encountered during the peak heat flux phase of re-entry. Therefore, the familiar Navier-Stokes-Fourier (NSF) equations are no longer valid because the gas is rarefied and the assumptions of the linear form of the constitutive relations for shear stress and heat flux no longer hold; transport of the fluid must be described by the Boltzmann equation, which for a single species, monatomic gas, has the form:

$$
\frac{\partial(n f)}{\partial t}+\mathbf{c} \frac{\partial(n f)}{\partial \mathbf{r}}+\mathbf{F} \frac{\partial(n f)}{\partial \mathbf{c}}=J\left(f, f^{*}\right)
$$


where $n f$ is the product of the number density and the normalised velocity distribution function, $\mathbf{r}$ and $\mathbf{c}$ are the position and velocity vectors of a molecule, respectively, $\mathbf{F}$ is an external force, $J\left(f, f^{*}\right)$ is a non-linear integral that describes the binary collisions, and the superscript $*$ represents post-collision properties. The collision integral takes the form:

$$
J\left(f, f^{*}\right)=\int_{-\infty}^{\infty} \int_{0}^{4 \pi} n^{2}\left(f^{*} f_{1}^{*}-f f_{1}\right) c_{r} \xi d \Omega d \mathbf{c}_{1},
$$

where $f$ and $f_{1}$ are the velocity distribution function at $\mathbf{c}$ and $\mathbf{c}_{1}$ respectively, $c_{r}$ is the relative speed of two colliding molecules, $\xi$ is the molecular crosssection, and $\Omega$ is the solid angle.

One of the dominant methods for finding a solution to the Boltzmann equation in the transition regime is the direct simulation Monte Carlo (DSMC) method [9]. DSMC is a particle based method, where the aim is to track a statistically representative number of particles in time and space, emulating the physics of the real gas. The first key assumption of the method is that a single DSMC simulator particle can represent any number of real gas atoms/molecules, which drastically reduces the computational expense of a simulation; for accuracy reasons, it is important to ensure that there are at least around 20 DSMC particles in a single computational cell [9]. Secondly, it is assumed that particle movements and collisions can be decoupled - collisions can be handled in a stochastic manner only after all particle movements have been dealt with deterministically. The method has now been used for 50 years [10] and has become the dominant numerical technique for solving gas flows that fall within the transition Knudsen number regime. It has been shown that the method provides an exact solution to the Boltzmann equation for a single species monatomic gas, in the limit of diminishing cell size 
and time step [11].

The DSMC algorithm is unconditionally stable, but there are two key numerical considerations that must be met in order to ensure that the results are reliable. Inter-particle collisions are restricted to a single computational cell: the local cell size must be smaller than the local mean free path to promote nearest-neighbour collisions and therefore a realistic transport of mass, momentum, and energy. Virtual sub-cells are often used to further promote nearest-neighbour collisions. A version of the dsmcFoam code [12], which has been modified by researchers at the University of Strathclyde, is used to perform all the numerical simulations in this paper.

In this paper, we investigate pressure-driven gas flow through two different ablative materials in both their virgin and pyrolised states; a commercially available cork-phenolic ablative material, hereafter called CPA-1, and a carbon fibre-phenolic ablative material, hereafter named CPA-2. The aim of the current work is develop a numerical framework that can be used to promote a better understanding of how the permeability of these materials changes from the virgin to the pyrolised states when gas flow through the materials is rarefied, which is possible both at the start and at the end of the high heat flux phase of an atmospheric entry, as shown in Reference [8]. The improved knowledge of the permeability will allow it to be employed with greater confidence in lower-order ablation models.

Work on rarefied gas flow through porous media using DSMC has received limited attention in recent years. de Socio and Marino [13] created an experimental set up consisting of glass spheres arranged randomly in a stainless steel cylinder, with a mean final porosity of 0.33 . Their experimen- 
tal sample was then subjected to a pressure differential with a nitrogen-fed flow-meter upstream and a vacuum pump downstream; the flow rate could be controlled manually. The pressure profile was measured at 7 static pressure holes along the length of the cylinder and compared to that from a modified direct simulation Monte Carlo method. The significant result from this work was showing that a Knudsen minimum [14] type phenomenon was found for the permeability of their experimental sample, i.e. there is a distinct minimum in permeability against gas rarefaction, with it being greater both below and above this critical Knudsen number. The DSMC method presented in the paper of de Socio and Marino [13] features a modified collision routine to emulate particles striking the surface of the packed spheres (the actual porosity is not physically modelled) and is only valid for a tortuosity that is characteristic of their experiment.

Oshima et al. [15] investigated the transport of gases through nano-scale channels that were filled with randomly generated spheres to a user-defined porosity. A pressure gradient was applied across the channel, with the length scales involved meaning that the Knudsen number is high. They measured the mass flux across the channels at different values of porosity, pressure gradient, and spherical particle diameters. Their results are qualitatively in agreement with Darcy's law [16] and it is demonstrated that the diameter of the particles has a significant impact on the permeability, even when the final porosity is the same, i.e. tortuosity is important.

Kalarakis et al. [17] created a physical representation of a porous microchannel geometry, where the blockage was created using a stochastic fractional Brownian technique. Nitrogen gas flows with an inlet-to-outlet 
pressure ratio of 2 were performed at different Knudsen numbers and the Klinkenberg effect is clearly found as the permeability increases as the Knudsen number is increased. It is found that the permeability can increase by 2 orders of magnitude in the range of the transition Knudsen number regime, showing how much the non-equilibrium effects can influence the fluid transport properties.

Firouzi and Wilcox [18] carried out molecular dynamics simulations of various gases through a porous carbon-based material. They found that permeability increases with porosity and that there is a critical porosity for their materials below which the permeability is zero due to poorly connected pores.

\section{Porosities, Numerical Meshing \& Simulation Parameters}

Micro-computed tomography $(\mu \mathrm{CT})$ scans of the CPA-1 and CPA-2 materials have been performed at the Austrian Foundry Research Institute (ÖGI) ${ }^{1}$, and the details of each scan are displayed in Table 1 . The scans have been performed on both materials in their virgin state, and after each has been pyrolised at conditions corresponding to their intended operating environments, i.e. the CPA-2 material has been pyrolised at $2000{ }^{\circ} \mathrm{C}$ and the CPA- 1 at $1300^{\circ} \mathrm{C}$.

\footnotetext{
${ }^{1}$ www.ogi.at/en/institut/oegi.php (Accessed 9th February 2015)
} 


\begin{tabular}{ccc}
\hline Material & nVoxels & Resolution $(\mu m)$ \\
\hline Virgin CPA-1 & $1573 \times 1554 \times 996$ & 3.411 \\
Virgin CPA-2 & $1500 \times 1500 \times 1000$ & 5.21 \\
Pyrolised CPA-1 & $1000 \times 1000 \times 1000$ & 6.111 \\
Pyrolised CPA-2 & $1000 \times 1000 \times 1000$ & 6.111 \\
\hline
\end{tabular}

Table 1: Details of the CT scans used in the current work.

In order to build numerical meshes that are a realistic representation of the materials, it is first important to determine the porosities of the samples. The CT scans give density values on a per voxel (where a voxel is a small volume, analogous to a pixel in 2D) basis, but the scale does not represent the physical density of the material at that point; it can only inform a user of the relative densities of each voxel region. A trial and error procedure was used to remove voxels with the density of air, which left voxels that represent the ablative material and the drinking straw it was contained in during the scanning process. This procedure involved finding a threshold value of voxel density which removed the region of air outside of the drinking straw material, but did not remove any of the straw or ablative material. This threshold value was different for each $\mu \mathrm{CT}$ scan because the scale does not represent real densities and is unique to each scan. A typical example of this is shown in Figure 2. The CPA-2 material is displayed in red, with the drinking straw in blue. The straw has an outer diameter of roughly $4.7 \mathrm{~mm}$. 


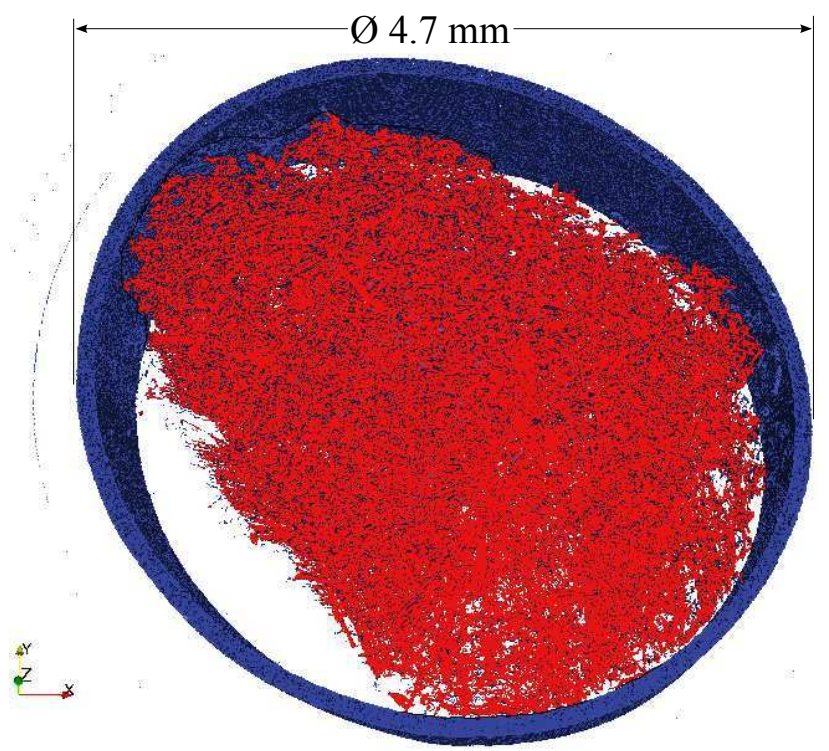

Figure 2: $\mu \mathrm{CT}$ scan of the pyrolised CPA-2 material with the voxels representing air removed. The CPA-2 material is shown in red, with the drinking straw container in blue.

The porosity of each material was then determined by extracting regions of increasing size from the geometrical centre of the samples. From Figure 2 it is clear that there are void regions between the straw and material. These are not porosity and were introduced because the material could not be machined to the exact shape of the straw, so care was taken to ensure the extracted volume contained only the ablative material, not regions of the straw or the empty volume between the straw and material. The final value for each material was considered to be that at which the porosity becomes roughly constant as the sample size increases, as shown in Figure 3. Each material was scanned at three different resolutions; low, standard, and high. The high resolution scans are used when possible in this work; a $\mu \mathrm{CT}$ scan at the standard resolution, but of a greater physical size, was included in 
Figure 3(b) to ensure that a reliable value of porosity was derived for that specific material. It has not been possible, due to the nature that particular high resolution scan, to derive a settled value of porosity, so the porosity from the standard resolution scan has been assumed to be the same as that which would be achieved from the high resolution scan. Table 2 contains the final values of porosity that have been used in the remainder of this work.

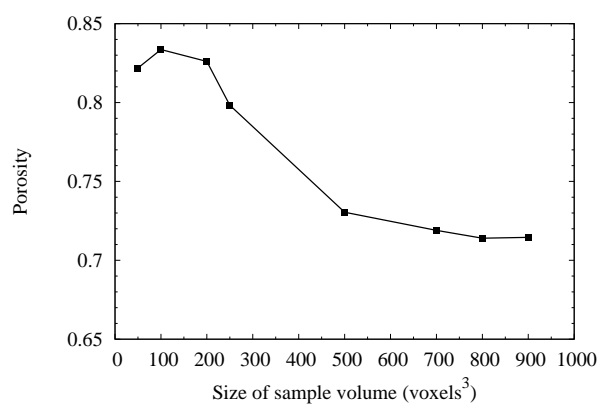

(a)

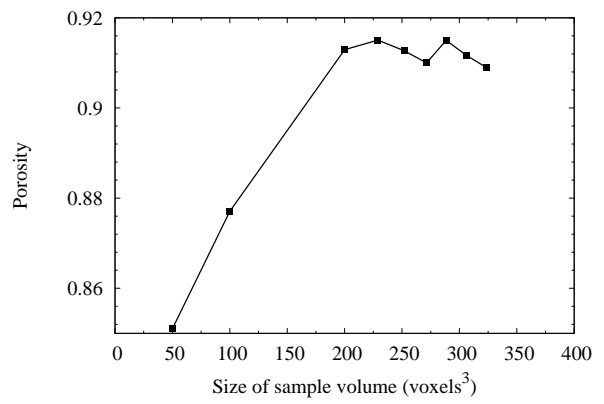

(c)

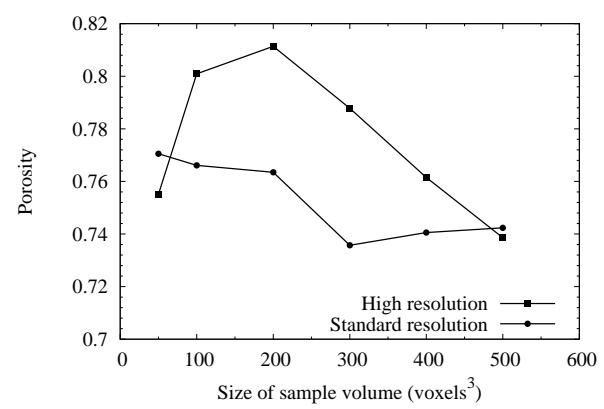

(b)

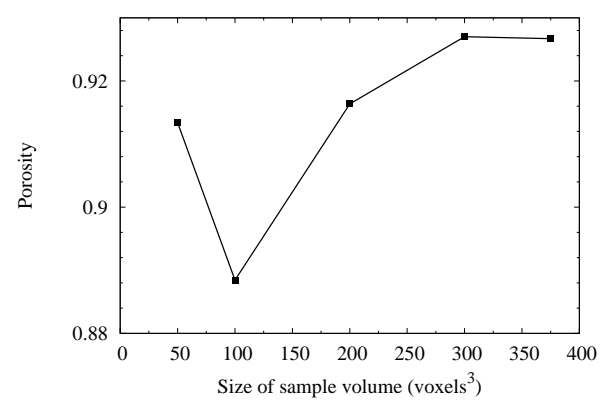

(d)

Figure 3: Porosities of the materials: (a) virgin CPA-1 material, (b) virgin CPA-2 material, (c) pyrolised CPA-1 material, and (d) pyrolised CPA-2 material. 


\begin{tabular}{cc}
\hline Material & Porosity \\
\hline Virgin CPA-1 & 0.714 \\
Virgin CPA-2 & 0.742 \\
Pyrolised CPA-1 & 0.909 \\
Pyrolised CPA-2 & 0.927 \\
\hline
\end{tabular}

Table 2: Details of the CT scans used in the current work.

The results show that the carbon-phenolic material is more porous than the cork-phenolic and as expected, both materials become significantly more porous after the resin has been burned off.

With the porosity of each material known, regions of size $50 \times 50 \times$ 50 voxels with a porosity equal to that displayed in Table 2 were identified and exported. To ensure that the permeability returned from such a small sample was reliable, regions of $100 \times 100 \times 100$ voxels with have also been extracted with the same porosity, in order to compare the results. Larger sample sizes were not considered in the current work due to restrictions with the computational expense of DSMC.

Once the $50^{3}$ and $100^{3}$ voxel regions were extracted, they were used to create numerical meshes within the OpenFOAM ${ }^{2}$ software. OpenFOAM is a $\mathrm{C}++$, open-source fluid dynamics toolbox and one of its key features is snappyHexMesh, an automatic meshing algorithm for use with complex 3D structures. A background mesh of cubic cells with the dimensions of the voxels described in Table 1 was created. Using the exported 3D geometry,

\footnotetext{
${ }^{2}$ www.openfoam.com (Accessed 9th February 2015)
} 
snappyHexMesh then automatically removed cells that corresponded to solid material, leaving behind a mesh that includes all of the internal paths that the gas could flow through within the sample. An example of the mesh for the pyrolised CPA-2 material is shown in Figure 4. The physical sizes of each

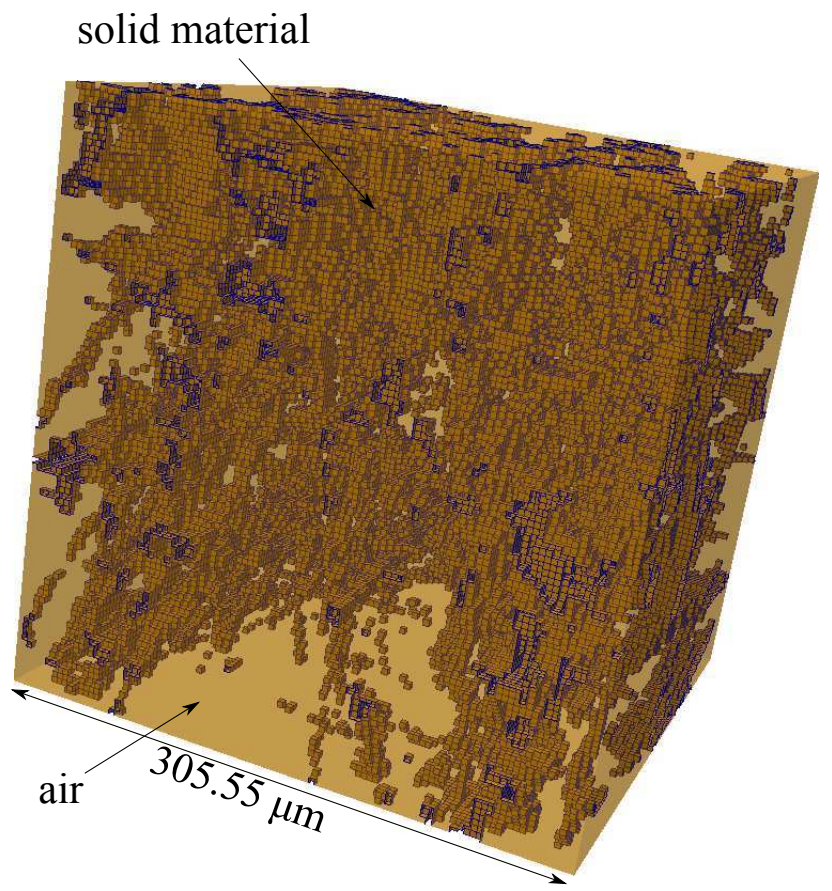

Figure 4: Screenshot of the numerical mesh created for the $100^{3}$ voxels pyrolised CPA-2. The opaque areas are regions of air and the solid surfaces are the charred carbon material.

mesh are listed in Table 3 and are the number of voxels multiplied by the resolution of the $\mu \mathrm{CT}$ scan. 


\begin{tabular}{cc}
\hline Mesh & Size $(\mu \mathrm{m})$ \\
\hline Small virgin CPA-1 & $170.55 \times 170.55 \times 170.55$ \\
Small virgin CPA-2 & $260.5 \times 260.5 \times 260.5$ \\
Large virgin CPA-1 & $341.1 \times 341.1 \times 341.1$ \\
Large virgin CPA-2 & $521 \times 521 \times 521$ \\
\hline Small pyrolised CPA-1 & $305.55 \times 305.55 \times 305.55$ \\
Small pyrolised CPA-2 & $305.55 \times 305.55 \times 305.55$ \\
Large pyrolised CPA-1 & $611.1 \times 611.1 \times 611.1$ \\
Large pyrolised CPA-2 & $611.1 \times 611.1 \times 611.1$ \\
\hline
\end{tabular}

Table 3: Physical size of each mesh for the DSMC simulations.

For each material, the inlets were considered, in separate simulations, to be in the $x-, y-$, and $z$-directions to help define the degree of anisotropy in the results. The remaining 4 outer boundaries were all set to be symmetry planes, so in effect an infinitely large block of the material was simulated, and the results were not influenced by the presence of the outer boundaries, other than the inlets and outlets. The solid material inside the mesh, as shown in Figure 4, were modelled as fully diffuse walls held at a temperature of $1500 \mathrm{~K}$.

The pyrolysis gas composition was chosen from Table 1 in Reference [19] at a temperature of $1500 \mathrm{~K}$; this temperature was chosen both because there is data available of pyrolysis gas composition for similar (carbon-phenolic) materials [19], and to be representative of the operating conditions of both materials. In addition, the CPA-2 material was pyrolised at $2273 \mathrm{~K}$ and the CPA-1 at $1573 \mathrm{~K}$, so this temperature can be representative of both materials. 
In reality, both materials would have different pyrolysis gas compositions and operate at different temperatures, but the aim of the current work is to show that this numerical procedure can be used to help better understand the material beaviour, so the choices of temperatures and gases are not considered critical here. The stagnation pressure and gas composition is detailed in Table 4. The gas may be chemically active, and so the influence of gas-gas chemical reactions was included, with all of the reactions detailed in Table 5 and Table 6. For the exchange reactions, the default activation energy $E_{a}$ for the forward reactions is the heat of reaction, and zero for the reverse reactions. Each reaction has been compared to an Arrhenius rate from the NIST database and Reference [20], and the dimensionless $a$ and $b$ parameters (which adjust the default activation energies) chosen both to match these rates and ensure that the ratio of the forward-to-reverse reactions remains consistent with statistical theory.

The reaction set is by no means complete; for example, we are restricting the current work to diatomic molecules and atoms. To the best of our knowledge, this is the first time that reactions involving carbon species have been considered using the quantum-kinetic $(\mathrm{QK})$ chemical reaction model [21].

In order to calculate the permeability, information on the gas viscosity is also required. For argon, the VHS viscosity index was used to calculate a viscosity of $8.42 \times 10^{-5} \mathrm{Nsm}^{-2}$. The pyrolysis gas is multi-species and composed of gases with widely different masses; the viscosity was estimated using the following relation [22], which takes into the account dissimilar masses on 
the efficiency of momentum transfer in a gas:

$$
\frac{1}{\mu}=\sum_{i=1}^{s}\left[\sum_{j=1}^{s}\left(\frac{y_{i} y_{j}}{\sqrt{\mu_{i}} \sqrt{\mu_{j}}} E_{i, j}^{\frac{1}{3}}\right)\right] \text {, }
$$

where

$$
E_{i, j}=\frac{2 \sqrt{m_{i}} \sqrt{m_{j}}}{m_{i}+m_{j}}
$$

and

$$
y_{i}=\frac{X_{i} \sqrt{M_{i}}}{\sum_{m=1}^{s}\left(X_{m} \sqrt{M_{m}}\right)} .
$$

In the above relations, $M_{i}$ is the molecular weight of species $i, m_{i}$ is the mass, and $X_{i}$ the mole fraction. Using equation (5), the viscosity of the pyrolysis gas mixture outlined in Table 4 was taken as $5.276 \times 10^{-5} \mathrm{Nsm}^{-2}$ in the present work.

\begin{tabular}{ccccccc}
\hline \multirow{2}{*}{$T(\mathrm{~K})$} & $P_{\text {out }}(\mathrm{Pa})$ & \multicolumn{5}{c}{ Mole Fraction } \\
\cline { 3 - 7 } & & $\mathrm{H}_{2}$ & $\mathrm{CO}$ & $\mathrm{N}_{2}$ & $\mathrm{NO}$ & $\mathrm{C}_{2} \mathrm{H}_{2}$ \\
\hline 1500 & 331.33 & 0.485 & 0.276 & 0.157 & 0.0522 & 0.0293 \\
\hline
\end{tabular}

Table 4: Pyrolysis gas parameters and composition used in the current work. 


\begin{tabular}{|c|c|}
\hline Reaction & Heat of Reaction $(\mathrm{K})$ \\
\hline $\mathrm{N}_{2}+M \rightarrow \mathrm{N}+\mathrm{N}+M$ & $-112,994$ \\
\hline $\mathrm{N}_{2}+A \rightarrow \mathrm{N}+\mathrm{N}+A$ & $-112,994$ \\
\hline $\mathrm{O}_{2}+M \rightarrow \mathrm{O}+\mathrm{O}+M$ & $-59,400$ \\
\hline $\mathrm{O}_{2}+A \rightarrow \mathrm{O}+\mathrm{O}+A$ & $-59,400$ \\
\hline $\mathrm{NO}+M \rightarrow \mathrm{N}+\mathrm{O}+M$ & $-75,544$ \\
\hline $\mathrm{NO}+A \rightarrow \mathrm{N}+\mathrm{O}+A$ & $-75,544$ \\
\hline $\mathrm{H}_{2}+M \rightarrow \mathrm{H}+\mathrm{H}+M$ & $-52,440$ \\
\hline $\mathrm{H}_{2}+A \rightarrow \mathrm{H}+\mathrm{H}+A$ & $-52,440$ \\
\hline $\mathrm{CO}+M \rightarrow \mathrm{C}+\mathrm{O}+M$ & $-128,929$ \\
\hline $\mathrm{CO}+A \rightarrow \mathrm{C}+\mathrm{O}+A$ & $-128,929$ \\
\hline $\mathrm{CN}+M \rightarrow \mathrm{C}+\mathrm{N}+M$ & $-107,199$ \\
\hline $\mathrm{CN}+A \rightarrow \mathrm{C}+\mathrm{N}+A$ & $-107,199$ \\
\hline
\end{tabular}

Table 5: Dissociation reactions considered in the current work.

\begin{tabular}{ccccccc}
\hline \multirow{2}{*}{ Reaction } & \multicolumn{2}{c}{ Activation Energy $(\mathrm{K})$} & \multicolumn{2}{c}{ Forward Coeffs. } & \multicolumn{2}{c}{ Reverse Coeffs. } \\
\cline { 2 - 7 } & Forward & Reverse & $a$ & $b$ & $a$ & $b$ \\
\hline $\mathrm{NO}+\mathrm{O} \leftrightarrow \mathrm{O}_{2}+\mathrm{N}$ & 19,700 & 0 & 0.085 & 0.65 & 0.1 & 0.1 \\
$\mathrm{~N}_{2}+\mathrm{O} \leftrightarrow \mathrm{NO}+\mathrm{N}$ & 37,500 & 0 & 0.15 & 0.15 & 0.033 & 0.8 \\
$\mathrm{~N}_{2}+\mathrm{C} \leftrightarrow \mathrm{CN}+\mathrm{N}$ & 23,200 & 0 & 0.08 & 0.25 & 0.05 & 0.8 \\
$\mathrm{CO}+\mathrm{O} \leftrightarrow \mathrm{O}_{2}+\mathrm{C}$ & 69,200 & 0 & 0.09 & 0.4 & 0.025 & 0.8 \\
$\mathrm{CN}+\mathrm{O} \leftrightarrow \mathrm{NO}+\mathrm{C}$ & 14,600 & 0 & 0.12 & 0.4 & 0.1 & 0.45 \\
\hline
\end{tabular}

Table 6: Exchange reactions considered in the current work. 


\begin{tabular}{cccccc}
\hline Species & $m\left(\times 10^{-27} \mathrm{~kg}\right)$ & $\omega$ & $d\left(\times 10^{-10} \mathrm{~m}\right)$ & $T_{\text {ref }}(\mathrm{K})$ & $\Theta_{v}(\mathrm{~K})$ \\
\hline $\mathrm{N}_{2}$ & 46.5 & 0.74 & 4.17 & 273 & 3371 \\
$\mathrm{O}_{2}$ & 53.12 & 0.77 & 4.07 & 273 & 2256 \\
$\mathrm{NO}$ & 49.88 & 0.79 & 4.20 & 273 & 2719 \\
$\mathrm{H}_{2}$ & 3.34 & 0.67 & 4.17 & 273 & 6159 \\
$\mathrm{CO}$ & 46.5 & 0.73 & 4.19 & 273 & 3122 \\
$\mathrm{CN}$ & 43.19 & 0.8 & 4.00 & 273 & 2981 \\
$\mathrm{~N}$ & 23.25 & 0.8 & 3.00 & 273 & - \\
$\mathrm{O}$ & 26.56 & 0.8 & 3.00 & 273 & - \\
$\mathrm{H}$ & 1.67 & 0.8 & 2.50 & 273 & - \\
$\mathrm{C}$ & 19.94 & 0.8 & 3.00 & 273 & - \\
$\mathrm{Ar}$ & 66.3 & 0.81 & 4.17 & 273 & - \\
\hline
\end{tabular}

Table 7: VHS parameters for the individual gas species [9].

Since the acetylene $\left(\mathrm{C}_{2} \mathrm{H}_{2}\right)$ is polyatomic and only $2.93 \%$ of the total gas composition, it was excluded from the DSMC simulations. The parameters used in the simulations for each species are detailed in Table 7, where $m$ is the molecular mass, $\omega$ is the viscosity exponent, $d$ is the molecular diameter, $T_{r e f}$ is the reference temperature for the viscosity model, and $\Theta_{v}$ is the characteristic vibrational temperature.

The outlet boundary conditions are fixed pressure outlets held at the chosen pressure of $331.33 \mathrm{~Pa}$. This is a very low pressure, but it designed to simulate the very early stages of re-entry, where the external flow is rarefied, and the material can still be considered near to its virgin state. For the pyrolised material, the same outlet pressure was used, which then represents 
a further point in the re-entry where the vehicle has decelerated significantly and the stagnation pressure has reduced again, as shown in Reference [8]. These represent points in the re-entry trajectory well before and after the peak heating point.

The outlet boundary condition used is described in Reference [23] and its dsmcFoam implementation has previously been validated in Reference [24] this boundary condition calculates the gas composition at the outlet from the number fractions in the cells attached to the outlet boundary, so can account for new species that are introduced by chemical reactions as the simulation proceeds. At the inlet, a defined inlet pressure boundary condition was used, as described in Reference [25]. As the simulation proceeds, this boundary condition works in a manner described in References $[23,24]$ to find velocities, temperatures, and densities at the boundaries that return the user defined pressure. The inlet temperature was set the same as for the solid material, $1500 \mathrm{~K}$.

Before performing simulations with the gas mixture outlined in Table 4, the simulations were first run with argon gas to have a benchmark to compare further results against. All of the simulations used the variable hard sphere (VHS) collision model [26] and the Larsen-Borgnakke phenomenological model [27] to redistribute energy between the rotational and translational modes. In $d s m c F o a m$, a pair-wise implementation of this redistribution model is used, with a constant relaxation probability of $1 / Z_{\text {rot }}$, where $Z_{\text {rot }}$ was set constant at 5 for all gas species in the current work. Vibrational energy is handled with a harmonic oscillator model and redistribution follows the algorithm outlined in Reference [28]. The simulations involving 
argon gas all used a constant timestep of $1 \times 10^{-9} \mathrm{~s}$, and to account for the smaller molecular mass of hydrogen, a timestep of $1 \times 10^{-10} \mathrm{~s}$ is used for all simulations with the pyrolysis gas.

An inlet-to-outlet pressure ratio $\left(P_{\text {in }} / P_{\text {out }}\right)$ of 2 was used for each case, with $P_{\text {in }}=331.33 \mathrm{~Pa}$. Using the same pressures for all cases ensures that the permeabilities are being measured at the same Knudsen numbers; this is required for a fair comparison as, unlike permeability to a liquid, it has been shown $[13,15]$ that the permeability to a gas is a function of rarefaction. The inlet Knudsen numbers for the cases with argon are 40.3 and 23.4 for the virgin CPA-1 and CPA-2, respectively. The mean free path $\lambda$ is taken as the VHS value, and the characteristic length $D$ as the size of a voxel in the CT scan (i.e. the scan resolution). Despite the large Knudsen numbers, these are not free-molecular flows: the average pore diameter is likely to be much larger than the scan resolution, but the scan resolution gives the smallest possible pore diameter. For both pyrolised materials, the Knudsen number is 22.5 .

The mass flow rate was monitored at multiple cross-sections and the simulation was considered converged once all of the measured mass flow rates were within $\pm 1 \%$ of each another. In addition, the number of particles in the system and the average linear kinetic energy is monitored to ensure a steady state solution has been reached before statistical sampling takes place. Sampling is performed in bins along the streamwise flow direction, with each bin covering up to $50 \times 50$ (for the small samples) and $100 \times 100$ (for the large samples) cells, depending on the local porosity. 
The simulations were run on the ARCHIE-WeSt supercomputer facility ${ }^{3}$ at the University of Strathclyde. The small sample sizes had around 2.5 million DSMC simulator particles at steady state and typically required around $250 \mathrm{CPU}$ hours to get the final results for both gases, while the larger simulations used 25 million particles and around 1700 CPU hours for the argon gas and $3360 \mathrm{CPU}$ hours (i.e. 70 hours on 48 cores) for the pyrolysis gas.

\section{Validation}

In order to validate this method for measuring the permeability of porous materials, a simulation was performed on a CT scan of Berea sandstone material [29]. Previous experimental studies of this material found its permeability to be between 0.48 Darcys [30] (using distilled water as the permeant) and 0.65 Darcys [31] $\left(1\right.$ Darcy $\left.=9.869233 \times 10^{-13} \mathrm{~m}^{2}\right)$. Complementary methods from the same authors, to determine the permeability of the same material found it analytically, based on caclulations of transport properties applied to scanning electron microscope scans, to be 0.56 Darcys [30], and 1.111-2.668 Darcys [31] using a numerical method to extract pore and throat networks and using these as an input in a pore-scale model.

The DSMC simulation here considers a $100 \times 100 \times 100$ voxel representation of the Berea sandstone, with a resolution of $5.345 \mu \mathrm{m}$. The porosity of the sample volume after removing voxels that represent air is 0.199. Argon gas was used as the permeant and, in order to minimise the Klinkenberg effects on the permeability, the pressures used are as high as possible with-

\footnotetext{
${ }^{3}$ www.archie-west.ac.uk (Accessed 9th February 2015)
} 
out making the simulation prohibitively computationally expensive. An inlet pressure and temperature of $2026.5 \mathrm{~Pa}$ and $300 \mathrm{~K}$ were used, respectively. The pressure gradient was applied in the $x$-direction, with an outlet pressure of $1013.25 \mathrm{~Pa}$, and the four remaining faces were symmetry planes. All solid surfaces were considered to reflect particles diffusely at $300 \mathrm{~K}$. The Knudsen number, based on the inlet pressure and the average pore radius previously calculated for this $\mu \mathrm{CT}$ scan [31], is 0.17 . The simulation was performed in parallel on $60 \mathrm{CPUs}$ and required a run time of 3.5 days to reach steady state and a further 2.5 days of sampling to reduce the statistical scatter in the measured mass flow rate. There were around 14 million DSMC particles at steady state and a timestep of $2 \times 10^{-9} \mathrm{~s}$ was used. Figure 5 shows the contours of pressure measured inside the pores of the Berea material.

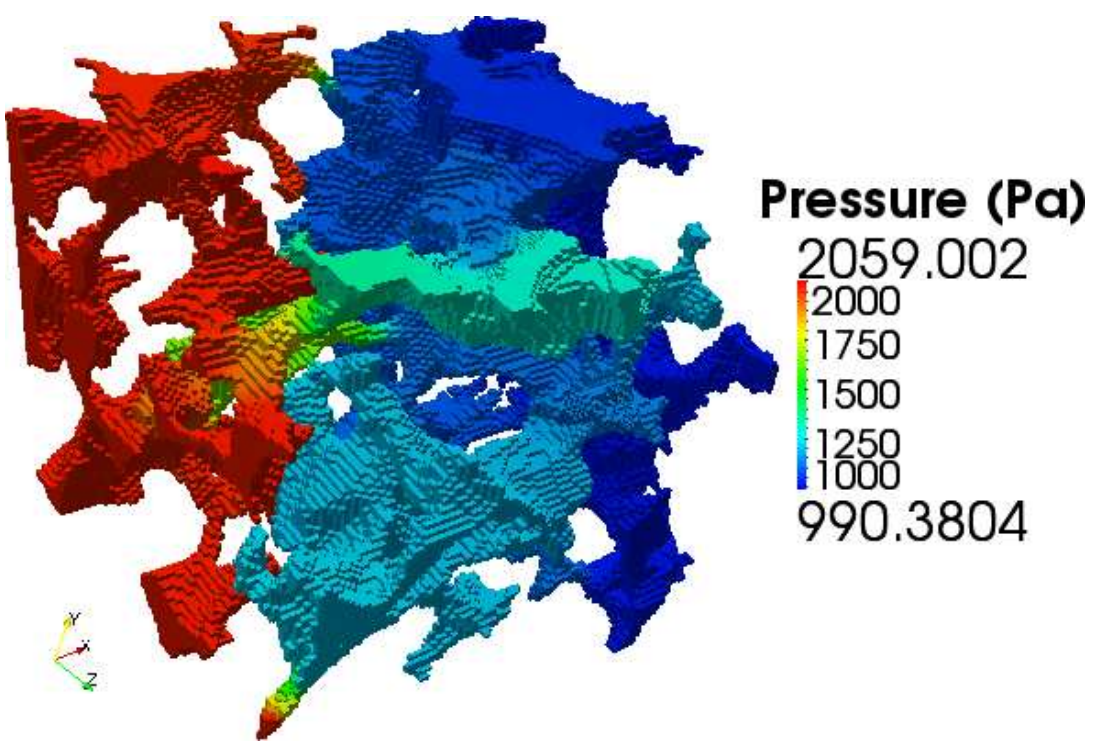

Figure 5: Contours of pressure in the Berea sandstone pores. 
The defined inlet and outlet pressures, $P_{\text {in }}$ and $P_{\text {out }}$, and the measured mass flow rate, were used with Equation (1) to define the permeability of the sample. The outlet volume flow rate $\dot{Q}$ is given from:

$$
\dot{Q}=\frac{\dot{m}}{\rho_{\text {out }}},
$$

where $\dot{m}$ is the mass flow rate in the system and $\rho_{\text {out }}$ is the mass density of the gas at the outlet.

From the exit volumetric flow rate, the DSMC simulation gives a permeability of 0.54 Darcys. It is important to note that this value of permeability is only valid for these specific conditions and that no attempt to calculate the rarefied permeability function has been made here. The calculated permeability compares well with the two experimental values stated above, even accounting for the fact that any Klinkenberg effects present may have increased the permeability from the DSMC simulation, particularly compared to the experimental value of 0.48 Darcys obtained using water. This result confirms that the current method for determining permeability from DSMC simulations can return realistic values, but also that the computational effort required to obtain them can be high.

\section{Results}

No chemical reactions occurred in any of the simulations at $1500 \mathrm{~K}$ with the pyrolysis gas mixture. Since nitrogen, in particular, has a relatively large characteristic dissociation temperature, this is not a surprising result and shows that gas phase chemical reactions are not likely to be an important factor in calculating the permeability of these materials. 
The normalised average cross-sectional pressure profiles in the stream direction for four of the simulations are shown in Figure 6. It is clear that all simulations have the same inlet and outlet pressures. The pressure profiles deviate from the simple straight line that would be expected for a continuum gas flow, but they are also significantly different from the well known nonlinear pressure profiles in transition regime gas flows [32-34]. 


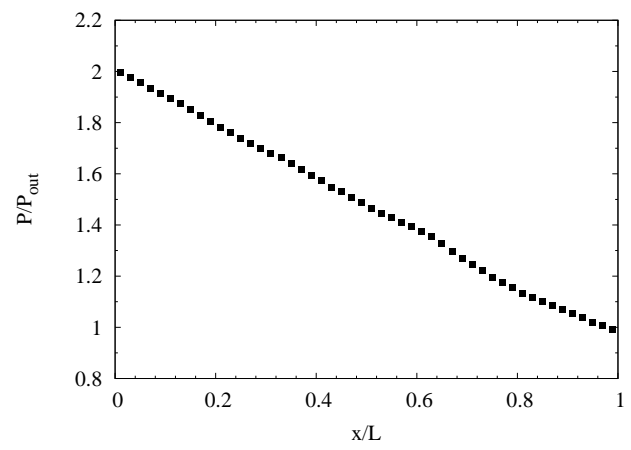

(a)

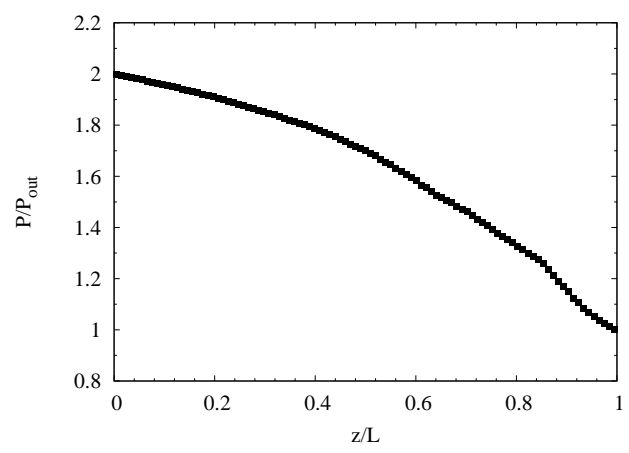

(c)

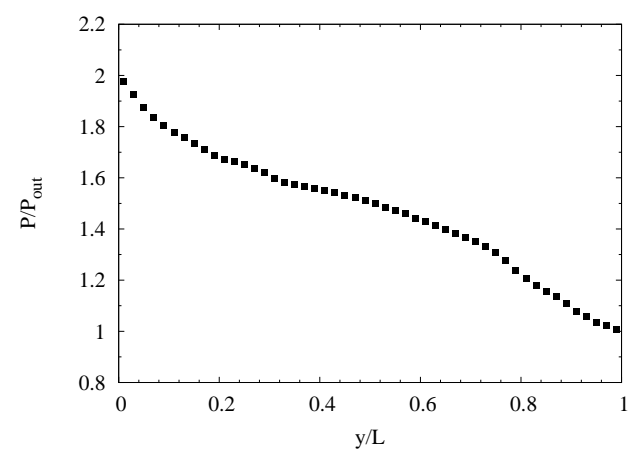

(b)

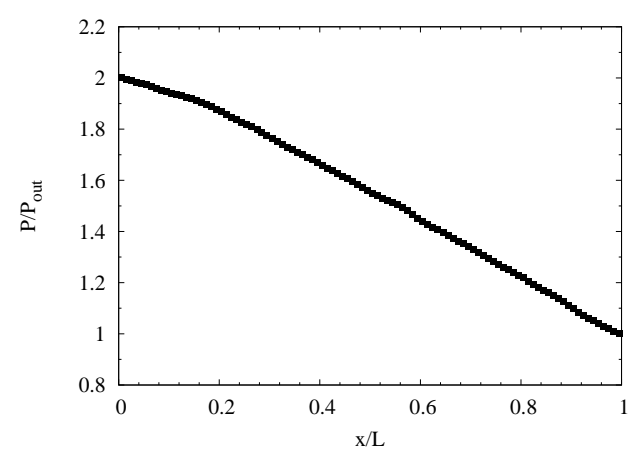

(d)

Figure 6: Average cross-sectional normalised pressure profiles for (a) argon gas $x$-direction flow through the small pyrolised CPA-2 material sample, (b) pyrolysis gas $y$-direction flow through the small virgin CPA-1 material, (c) pyrolysis gas $z$-direction flow through the large virgin CPA-1 material, and (d) argon gas $x$-direction flow through the large pyrolised CPA-2 material.

Figure 7 shows the average cross-sectional streamwise Mach number profiles through four of the samples. The Mach number magnitude increases from inlet to outlet for all cases as expected, with greater Mach numbers being reached in the flow through the pyrolised materials. This is expected because the porosity of the pyrolised samples is greater than the virgin. In 
addition, the Mach number reached in the small pyrolised sample in Figure 7 (a) is greater than that of the large sample in Figure 7 (d) because the pressure gradient is greater in the small samples, leading to a larger driving force. The scattered nature of the profiles is not noise; the statistical uncertainties have been calculated [35] as fractions of a percent. Taking the cross-sectional average of the profile reduces the scatter to an acceptable level by including a large number of cells (typically around 8,000 for the large samples depending on the local porosity) in the measurement volume for each bin, giving a minimum of 32000 particles in a single bin. Rather, the scatter is due to the tortuous path that the gas follows through the material's pores. 


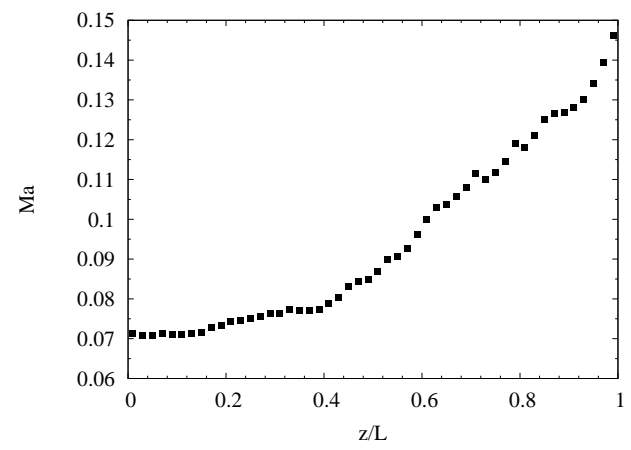

(a)

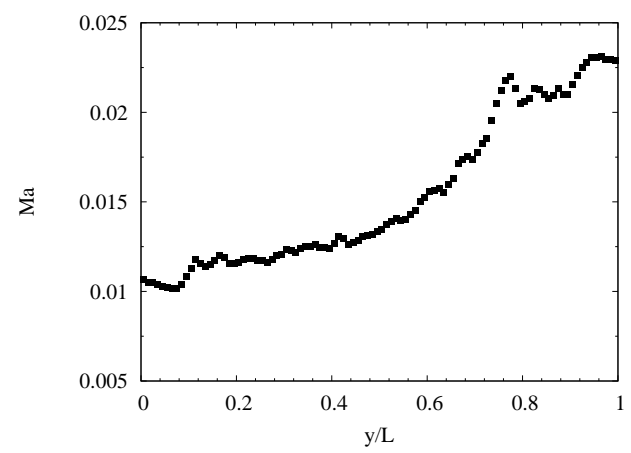

(c)

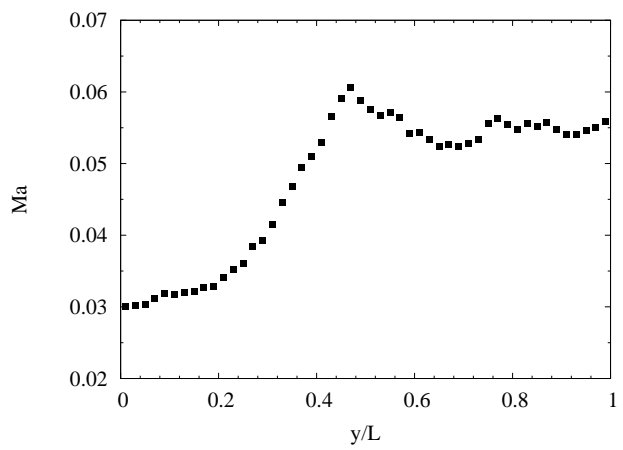

(b)

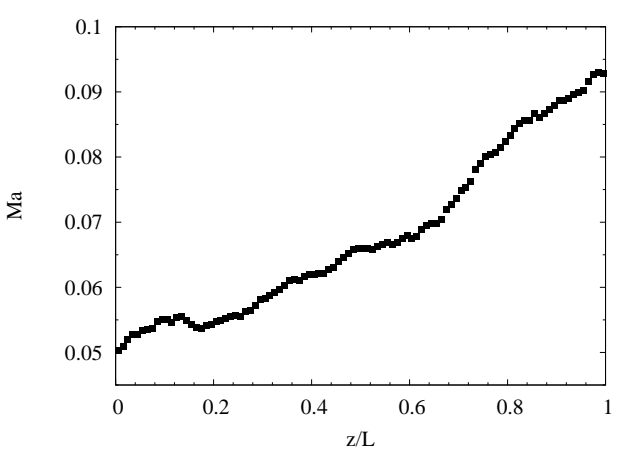

(d)

Figure 7: Average cross-sectional streamwise Mach number profiles for (a) pyrolysis gas $z$-direction flow through the small pyrolised CPA-1 material, (b) argon gas $x$-direction flow through the small virgin CPA-2 sample, and (c) argon gas $y$-direction flow through the large virgin CPA-2 material sample, and (d) pyrolysis gas $z$-direction flow through the large pyrolsied CPA-1 material sample. 


\begin{tabular}{cccc}
\hline \multirow{2}{*}{ Sample } & & \multicolumn{2}{c}{ Permeability $\left(\mathrm{m}^{2}\right)$} \\
\cline { 2 - 3 } & & Argon & Pyrolysis Gas \\
\hline \multirow{3}{*}{ Small virgin CPA-2 } & $y$ & $1.34 \times 10^{-9}$ & $1.43 \times 10^{-9}$ \\
& $z$ & $1.12 \times 10^{-9}$ & $1.18 \times 10^{-9}$ \\
\hline & $x$ & $8.91 \times 10^{-10}$ & $8.89 \times 10^{-10}$ \\
Large virgin CPA-2 & $y$ & $5.14 \times 10^{-10}$ & $5.32 \times 10^{-10}$ \\
& $z$ & $1.20 \times 10^{-9}$ & $1.32 \times 10^{-9}$ \\
\hline & $x$ & $9.89 \times 10^{-9}$ & $1.14 \times 10^{-8}$ \\
Small pyrolised CPA-2 & $y$ & $5.36 \times 10^{-9}$ & $6.23 \times 10^{-9}$ \\
& $z$ & $6.43 \times 10^{-9}$ & $7.20 \times 10^{-9}$ \\
\hline & $x$ & $5.48 \times 10^{-9}$ & $6.10 \times 10^{-9}$ \\
Large pyrolised CPA-2 & $y$ & $4.62 \times 10^{-9}$ & $5.04 \times 10^{-9}$ \\
& $z$ & $4.06 \times 10^{-9}$ & $4.41 \times 10^{-9}$ \\
\hline
\end{tabular}

Table 8: Calculated permeability values for the CPA-2 samples.

The permeabilities of each material to both argon and the gas mixture were calculated using Equation (1). Table 8 contains the results of permeability for the CPA-2 material samples. Firstly, considering only the argon gas results, the permeability of the pyrolised material is significantly larger than that of the virgin material, because its porosity is greater, and so transport of a gas through the material is easier than in the corresponding virgin material. The permeability in the $y$-direction of the large virgin CPA-2 sample has been found to be much smaller than in the other two directions. This anisotropic permeability could be due to the carbon fibres being laid out in a 
regular pattern during manufacture, or perhaps preferential alignment of the fibres at the micro and nano scale. This was not found in the smaller sample size, although the larger sample sizes likely give a more accurate value of the permeability as they can capture a geometry that is more representative of the $\mu \mathrm{CT}$ scan. Ideally, simulations on even larger sample sizes would be performed, but the relatively expensive numerical nature of the DSMC method has prevented this in the current work.

It is found that the permeability of the samples to argon is smaller compared to that with pyrolysis gas, despite using the same inlet pressure and pressure gradient. Although the current work does not investigate the presence of the Klinkenberg effect in these materials, this result is consistent with the Klinkenberg effect, where the permeability is given from Equation (9),

$$
k=k_{0}\left(1+\frac{b}{P}\right),
$$

where $k_{0}$ is the permeability of the material to a liquid, $P$ is the pore pressure, and $b$ is a slip correction factor that is a function of gas rarefaction. The value of $b$ will vary between argon and the gas mixture; there is a significant molar fraction of hydrogen in the pyrolysis gas, which gives it a larger mean free path than the argon and could account for the increased permeability. However, it is important to note that these values of permeability (and those in Table 9) are only valid for these specific conditions and that no attempt to calculate the rarefied permeability function of Equation (9) has been made here. 


\begin{tabular}{|c|c|c|c|}
\hline \multirow{2}{*}{ Sample } & & \multicolumn{2}{|c|}{ Permeability $\left(\mathrm{m}^{2}\right)$} \\
\hline & & Argon & Pyrolysis Gas \\
\hline \multirow{3}{*}{ Small virgin CPA-1 } & $x$ & $4.10 \times 10^{-10}$ & $4.49 \times 10^{-10}$ \\
\hline & $y$ & $3.93 \times 10^{-10}$ & $4.32 \times 10^{-10}$ \\
\hline & $z$ & $3.73 \times 10^{-10}$ & $4.07 \times 10^{-10}$ \\
\hline \multirow{3}{*}{ Large virgin CPA-1 } & $x$ & $2.49 \times 10^{-10}$ & $2.35 \times 10^{-10}$ \\
\hline & $y$ & $2.83 \times 10^{-10}$ & $2.68 \times 10^{-10}$ \\
\hline & $z$ & $2.29 \times 10^{-10}$ & $2.16 \times 10^{-10}$ \\
\hline \multirow{3}{*}{ Small pyrolised CPA-1 } & $x$ & $5.41 \times 10^{-9}$ & $6.02 \times 10^{-9}$ \\
\hline & $y$ & $2.76 \times 10^{-9}$ & $3.08 \times 10^{-9}$ \\
\hline & $z$ & $2.35 \times 10^{-9}$ & $2.62 \times 10^{-9}$ \\
\hline \multirow{3}{*}{ Large pyrolised CPA-1 } & $x$ & $3.68 \times 10^{-9}$ & $3.93 \times 10^{-9}$ \\
\hline & $y$ & $3.21 \times 10^{-9}$ & $3.40 \times 10^{-9}$ \\
\hline & $z$ & $2.73 \times 10^{-9}$ & $2.86 \times 10^{-9}$ \\
\hline
\end{tabular}

Table 9: Calculated permeability values for the CPA-1 samples.

The results for the CPA-1 material follow a similar trend to the CPA-2 results, with the pyrolised material having a much greater permeability than the virgin material. However, the large virgin CPA-1 results show a slightly lower permeability for pyrolysis gas compared to argon, which is in contrast the rest of the results in the current work.

\section{Conclusions}

A framework for identifying the porosity of a material from a $\mu \mathrm{CT}$ scan, identifying a suitable candidate sub-region, and creating a mesh for DSMC 
simulations was established. This allows for the flow of rarefied gases through a porous heat shield material to be investigated using a physically realistic geometry. The problems with the size limits imposed by the computational expense of the numerical method were discussed, and a reasonable attempt to overcome them and obtain meaningful results from smaller geometries was described. The porosities of both CPA-2 and CPA-1 were then calculated in their virgin and pyrolised forms, with the porosity significantly increasing once the material has been pyrolised.

The permeability of a Berea sandstone sample was calculated, in order to validate the permeabilities of the ablative materials. The value of 0.54 Darcys is in good agreement with published experimental data and is in fact more reliable than some previous attempts to numerically determine the permeability of Berea sandstone. The results for the permeability of the ablative materials show some expected behaviour; the pyrolised material samples are more permeable because their porosities are greater, and so transport of a gas through the material is easier than in the corresponding virgin material. The CPA-1 becomes around ten times more permeable in its pyrolised form compared to its virgin form. The CPA-2 also becomes significantly more permeable, but not to the same extent: it becomes around five times more permeable according to the current results.

It is found that the permeability of the samples to argon is smaller compared to that with pyrolysis gas, despite using the same inlet pressure and pressure gradient. Although the current work does not investigate the presence of the Klinkenberg effect in these materials, this does show the importance of choosing a representative gas when performing permeability studies 
that include rarefied gases.

The current analysis is restricted only to gas-phase chemical reactions and those employed in this work have not had an affect on the permeability values, but for other gas combinations there may be reactions with lower activation energies, so gas-phase chemical reactions should not be neglected. The presence of gas-surface chemical reactions has not been investigated here and may be an important factor.

\section{Acknowledgements}

This work was supported by the EU/FP7 ABLAMOD program, grant no. FP7-SPACE-2012-312987. Results were obtained using the EPSRC funded ARCHIE-WeSt High Performance Computer (www.archie-west.ac.uk). EPSRC grant no. EP/K000586/1. The authors wish to thank Erhard Kaschnitz of the Austrian Foundry Research Institute (ÖGI) who performed the CT scans of the materials as part of the ABLAMOD program.

\section{References}

[1] D. E. Brownlee, P. Tsou, J. D. Anderson, M. S. Hanner, R. L. Newburn, Z. Sekanina, B. C. Clark, F. Hrz, M. E. Zolensky, J. Kissel, J. A. M. McDonnell, S. A. Sandford, and A. J. Tuzzolino. Stardust: Comet and interstellar dust sample return mission. Journal of Geophysical Research: Planets, 108(E10):1-15, 2003.

[2] H. K. Tran, C. E. Johnson, D. J. Rasky, F. C. L. Hui, M.-T. Hsu, T. Chen, Y. K. Chen, D. Paragas, and L Kobayashi. Phenolic im- 
pregnated carbon ablators (PICA) as thermal protection systems for discovery missions. Technical Report TM 110440, NASA, 1997.

[3] H. Weng and A. Martin. Multidimensional modeling of pyrolysis gas transport inside charring ablative materials. Journal of Thermophysics and Heat Transfer, 28(4):583-597., 2014.

[4] W. Tanikawa and T. Shimamoto. Klinkenberg effect for gas permeability and its comparison to water permeability for porous sedimentary rocks. Hydrology and Earth System Sciences Discussions, 3(4):13151338, 2006.

[5] L. J. Klinkenberg. The permeability of porous media to liquid and gases. Drilling and Production Practice, American Petroleum Inst., pages 200213, 1941.

[6] J.C. Maxwell. On stresses in rarefied gases arising from the inequalities of temperature. Phil. Trans. R. Soc. A, 170:231-256, 1879.

[7] M. Gad-el-Hak. The fluid mechanics of microdevices - the Freeman Scholar lecture. J. Fluids Eng., 121(1):5-33, 1999.

[8] J. Lachaud, I. Cozmuta, and N. N. Mansour. Multiscale approach to ablation modeling of phenolic impregnated carbon ablators. Journal of Spacecraft and Rockets, 47(6):910-921, 2010.

[9] G.A. Bird. Molecular Gas Dynamics and the Direct Simulation of Gas Flows. Oxford Science Publications, Oxford University Press Inc, New York, 1994. 
[10] G.A. Bird. Approach to translational equilibrium in a rigid sphere gas. Physics of Fluids, 6(10):1518-1519, 1963.

[11] W. Wagner. A convergence proof for Bird's direct simulation Monte Carlo method for the Boltzmann equation. Journal of Statistical Physics, 66:1011-1044, 1992.

[12] T. J. Scanlon, E. Roohi, C. White, M. Darbandi, and J.M. Reese. An open source, parallel DSMC code for rarefied gas flows in arbitrary geometries. Computers $\&$ Fluids, 39(10):2078-2089, 2010.

[13] L. M. de Socio and L. Marino. Gas flow in a permeable medium. Journal of Fluid Mechanics, 557:119-133, 2006.

[14] W. Steckelmacher. Knudsen flow 75 years on: the current state of the art for flow of rarefied gases in tubes and systems. Reports on Progress in Physics, 49(10):1083-1107, 1986.

[15] T. Oshima, S. Yonemura, and T. Tokumasu. A numerical study for transport phenomena of nanoscale gas flow in porous media. AIP Conference Proceedings, 1501(1):809-815, 2012.

[16] S. Whitaker. Flow in porous media I: A theoretical derivation of Darcy's law. Transport in Porous Media, 1(1):3-25, 1986.

[17] A.N. Kalarakis, V.K. Michalis, E.D. Skouras, and V.N. Burganos. Mesoscopic simulation of rarefied flow in narrow channels and porous media. Transport in Porous Media, 94(1):385-398, 2012. 
[18] M. Firouzi and J. Wilcox. Molecular modeling of carbon dioxide transport and storage in porous carbon-based materials. Microporous and Mesoporous Materials, 158:195-203, 2012.

[19] A. Martin and I.D. Boyd. Chemistry model for ablating carbon-phenolic material during atmospheric re-entry. In 48th AIAA Aerospace Sciences Meeting Including the New Horizons Forum and Aerospace Exposition, 2010.

[20] E. Farbar, I.D. Boyd, and A. Martin. Modeling ablation of charring heat shield materials for non-continuum hypersonic flow. In 50th AIAA Aerospace Sciences Meeting including the New Horizons Forum and Aerospace Exposition, 2012.

[21] G.A. Bird. Chemical reactions in DSMC. In Rarefied Gas Dynamics 2010, 2010.

[22] T.A. Davidson. A simple and accurate method for calculating viscosity of gaseous mixtures. U.S. Department of the Interior, Bureau of Mines, 1993.

[23] W.W. Liou and Y. Fang. Implicit boundary conditions for direct simulation Monte Carlo method in MEMS flow predictions. CMES: Computer Modeling in Engineering \&3 Sciences, 1(4):119-128, 2000.

[24] C. White, M.K. Borg, T.J. Scanlon, and J.M. Reese. A DSMC investigation of gas flows in micro-channels with bends. Computers 83 Fluids, 71:261-271, 2013. 
[25] M. Wang and Z. Li. Simulations for gas flows in microgeometries using the direct simulation Monte Carlo method. International Journal of Heat and Fluid Flow, 25(6):975-985, 2004.

[26] G.A. Bird. Definition of mean free path for real gases. Physics of Fluids, 26(11):3222-3223, 1983.

[27] C. Borgnakke and P.S. Larsen. Statistical collision model for Monte Carlo simulation of polyatomic gas mixture. Journal of Computational Physics, 18(4):405-420, 1975.

[28] G.A. Bird. A comparison of collision energy-based and temperaturebased procedures in DSMC. In Rarefied Gas Dynamics 2008, 2008.

[29] H. Dong and M. Blunt. Pore-network extraction from microcomputerized-tomography images. Physical Review E, 80:036307, 2009.

[30] E. M. Schlueter. Predicting the Transport Properties of Sedimentary Rocks From Microstructure. PhD thesis, Lawrence Berekely Laboratory: University of California, 1995.

[31] H. Dong. Micro-CT Imaging and Pore Network Extraction. PhD thesis, Imperial College London, 2007.

[32] W.W. Liou and Y. Fang. Microfluid Mechanics. McGraw-Hill, Two Penn Plaza, New York, 2006.

[33] Ehsan Roohi, Masoud Darbandi, and Vahid Mirjalili. Direct simulation Monte Carlo solution of subsonic flow through micro/nanoscale channels. Journal of Heat Transfer, 131(9):092402, 2009. 
[34] C. Cai, Q. Sun, and I.D. Boyd. Gas flows in microchannels and microtubes. Journal of Fluid Mechanics, 589(-1):305-314, 2007.

[35] N G Hadjiconstantinou, A L Garcia, M Z Bazant, and G He. Statistical error in particle simulations of hydrodynamic phenomena. J. Comput. Phys., 187(1):274-297, 2003. 